\title{
Computational characterization of an axial rotor fan
}

\author{
Unai Fernandez-Gamiz \\ University of the Basque Country (UPV/EHU), Faculty of Engineering of Vitoria-Gasteiz, Department of Nuclear \\ Engineering and Fluid Mechanics, C/Nieves Cano 12, 01006, Vitoria-Gasteiz, Spain, unai.fernandez@ehu.es, \\ orcid.org/0000-0001-9194-2009 \\ Mustafa Demirci \\ Gazi University, Department of Energy Systems Engineering, Faculty of Technology, Ankara, Turkey, \\ mdemirci66@ gmail.com,orcid.org/0000-0002-7050-8323 \\ Mustafa İlbaş \\ Gazi University, Department of Energy Systems Engineering, Faculty of Technology, Ankara, Turkey, \\ ilbas@gazi.edu.tr, orcid.org/0000-0001-6668-1484 \\ Ekaitz Zulueta
}

University of the Basque Country (UPV/EHU), Faculty of Engineering of Vitoria-Gasteiz, Department of Systems Engineering and Automatic Department, C/Nieves Cano 12, 01006, Vitoria-Gasteiz, Spain, ekaitz.zulueta@ehu.eus, orcid.org/0000-0001-6062-9343

Jose Antonio Ramos

University of the Basque Country (UPV/EHU), Faculty of Engineering of Vitoria-Gasteiz, Department of Electrical Engineering, C/Nieves Cano 12, 01006, Vitoria-Gasteiz, Spain, josean.ramos@ehu.es ; orcid.org/0000-0001-9706-4016 Jose Manuel Lopez-Guede

University of the Basque Country (UPV/EHU), Faculty of Engineering of Vitoria-Gasteiz, Department of Electrical Engineering, C/Nieves Cano 12, 01006, Vitoria-Gasteiz, Spain, jm.lopez@ehu.es, orcid.org/0000-0002-5310-1601 Erol Kurt

Gazi University, Department of Electrical and Electronics Engineering, Faculty of Technology, Ankara, Turkey, ekurt52tr@yahoo.com,orcid.org/0000-0002-3615-6926

Arrived: 26.10.2017 Accepted: 28.12.2017 Published: 29.12.2017

\begin{tabular}{|c|c|}
\hline & $\begin{array}{l}\text { Axial flow fans are broadly applied in numerous industrial applications because of their } \\
\text { simplicity, compactness and moderately low cost, such us propulsion machines and cooling } \\
\text { systems. Computational fluid dynamics techniques are commonly applied to investigate flow } \\
\text { phenomena through the axial fan and the rotor dynamic performance. In the present work, a } \\
\text { computational model of an axial fan is presented in the current study. Numerical simulations of a } \\
\text { single stage axial fan on variable conditions have been performed to obtain the detailed flow field } \\
\text { of the centrifugal fan. The investigation of the current work is focused on the rotor-stator } \\
\text { configuration and the modeling of aerodynamic behavior of the blade rows. The precise } \\
\text { prediction of axial force and efficiency has essential implication for the optimized operation of } \\
\text { axial fan and the choice of thrust bearing. Furthermore, it can act as guide for the geometrical and } \\
\text { structural axial fan design and the study of axial force prediction. }\end{array}$ \\
\hline Keywords: & Axial fan, Computational fluid dynamics, Rotor dynamics \\
\hline
\end{tabular}

Fernandez-Gamiz, U, Demirci, M, İlbaş, M, Zulueta, E, Ramos, JA, Lopez-Guede, JM,

Cite this paper as: $\quad$ Kurt E. Computational characterization of an axial rotor fan, Journal of Energy

Systems 2017; 1(4):129-137 DOI: 10.30521/jes.346660

(C) 2017 Published by peer-reviewed open access scientific journal, JES at DergiPark (www.dergipark.gov.tr/jes)

\begin{tabular}{r|l} 
Nomenclature & \\
\hline CFD & Computational Fluid Dynamics \\
NACA & National Advisory Committee for Aeronautics \\
BEP & Best Efficiency Point \\
ASME & American Society of Mechanical Engineers \\
VAWT & Vertical Axis Wind Turbines \\
ANN & Artificial Neural Network \\
AFPMSG & Axial Flux Permanent Magnet Synchronous Generator \\
\hline \hline
\end{tabular}




\section{INTRODUCTION}

Axial flow fans are widely used in many industrial applications ranging from cooling systems to the propulsion devices, to emphasize extreme scales. For better understanding of the flow phenomena through the axial fan and the rotor dynamic behavior, CFD techniques are commonly used and today almost unavoidable to include them in the machine design and testing. It is, however, essential to develop suitably accurate computational tools and validate them extensively. They are used in many industrial applications because of their simplicity, compactness and relatively low cost. They are applied from traditional cooling systems to the most innovative propulsion systems. Generally, axial flow machines have high specific velocity and low specific diameter on a single-stage basis. It means that the specific energy usually is quite small and the flow rate is relatively large [1].

For better understanding of the flow phenomena through the axial fan and the rotor dynamic behavior, CFD techniques are commonly used and today almost unavoidable to include them in the machine design and testing by Matai et al. [2]. It is however, essential to develop suitably accurate computational tools and validate them extensively, such as the comparison study of Prachar[1], where a comparison between CFD simulations and experimental results of an axial flow fan rotor was carried out with good agreement between both. In Ref. [3],the authors proposed several analytical formulas and charts to calculate the efficiency of different axial fan configurations on a tube. Matai et al [2] proposed quite a few design parameters to evaluate the performance of an axial fan using Computational Fluid Dynamics CFD techniques. In the studies of Gör et al [4] and Celik et al [5], an estimation study on the output power and the efficiency of a new-designed axial flux permanent magnet synchronous generator (AFPMSG) was performed and a multi-layer feedforward artificial neural network (ANN) is developed. Furthermore, Pelletier et al [6] and Yoon et al [7] explored three dimensional flow in a three-bladed rotor of an axial fun for aircraft applications. In this case, the rotor was modeled as an actuator disk. Matai et al [2] evaluated the effects of different design parameters on the aerodynamic performance of an axial fan by means of computational techniques. Mathematical models such as Twizell et al [8] have been also developed to empirically formulate the connection between the fan static pressure, the blade pitch angle and the volume in the inlet. According to [9], the performance parameters for a turbo machine are usually considered in terms of their efficiency, shaft power, energy exchange of the fluid (i.e. pressure drop), and fluid flow rate through the machine.

These previous performance parameters are related each other and to the machine operating parameters: Rotational velocity, fluid density and viscosity. The relationships among those parameters are the most important information for an optimal device design [10]. In this study, the sound/noise generated by the axial fan is not treated as performance variable. Usually, the machine performance experiments are done with utensils and procedures specifies by recognized standards to ensure the device accuracy, acceptance and the reproducibility of the test results [11]. For fans, the most suitable standard is the Laboratory Methods of Testing Fans for Ratings, [12]. Another option of field test can be found in ASME-PTC 11, [13].

There are several important things to notice from the performance curves of an axial fan. Firstly, the socalled best point of efficiency BEP, which is reached in the point of maximum efficiency in the curve defined by $\eta$ versus air flow Q (Fig. 1). BEP is defined by the corresponding values of volume flow rate $\mathrm{Q}$, pressure raise $\Delta \mathrm{p}$, shaft input power $\mathrm{P}_{\mathrm{sh}}$, and the machine noise $\mathrm{L}_{\mathrm{w}}$, as illustrated in Figure 1. In the right side of BEP, the variable of pressure raise $\Delta \mathrm{p}$ decreases as the volume flow rate $\mathrm{Q}$ increases, yielding a negative slope that represents a stable range of performance for the fan. In the left side of the $\mathrm{BEP}$ the pressure raise curve goes to zero slope condition. After that, the positive slope comes and it is the zone where the fan would operate unstably. 


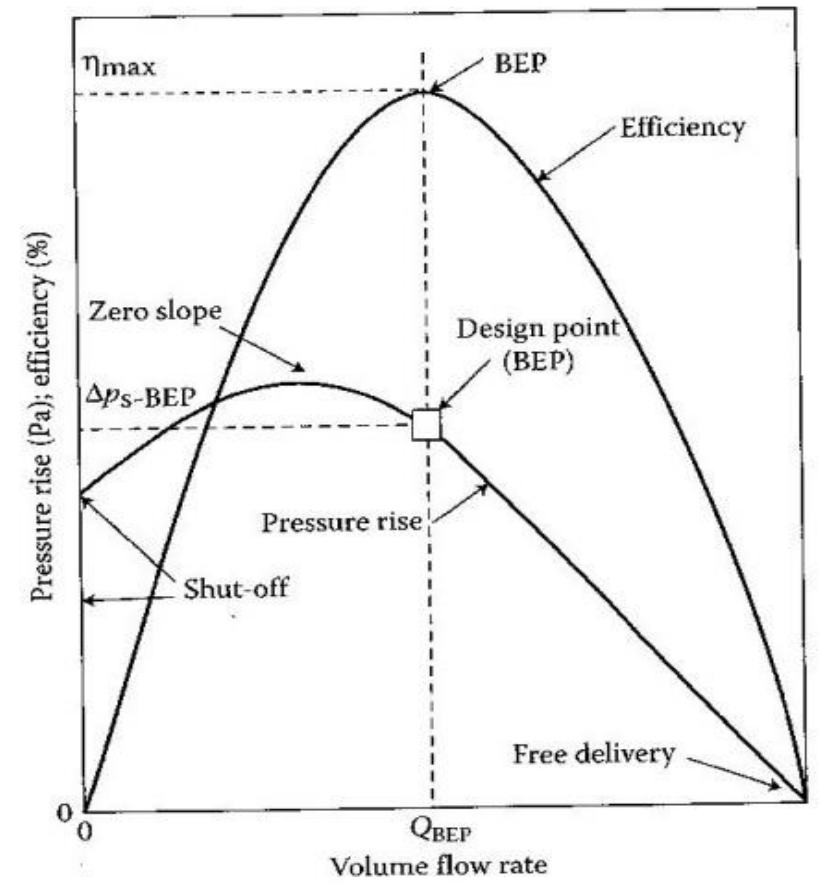

Figure 1. Pressure and efficiency curves [7].

The overall efficiency for an axial fan for incompressible flow can be calculated by Eq. (1):

$$
\eta_{T}=\frac{Q\left(\Delta p_{T}\right)}{P_{s h}}
$$

The dynamical behavior of an industrial axial fan has been simulated using CFD tools. A computational model of an axial fan rotor is presented in the current study, as illustrated in Figure 2. The reason of this work is to study the method and precision of simulating axial flow fans with three-dimensional CFD models. The results and knowledge gained will be used to improve currently used axial ventilators.

In the work of Castegnaro [14], the fan blade design was based on NACA65 series of airfoils and the damaging effects of the low Reynolds numbers on the blades performance have been reported in that manner. Carolus and co-workers[15] compared two fan rotors blade types based on NACA 4512 and FX60-126 airfoil designs with satisfactory results for NACA based designs. S-type blades with symmetric NACA based airfoils have been also used for reverse axial flow fans, such as in the study of Diangui [16].

\section{COMPUTATIONAL SETTINGS}

In the present work, the aerodynamic performance of an axial fan rotor shown in Fig. 2 has been investigated. The rotor has been designed with 30 blades inclined an angle to the oncoming flow and their geometry is based on the traditional NACA airfoils to achieve high efficiency. Fig. 3 shows a detailed view of the blade chord length and inclination respect to the rotor disc. The diameter of the rotor is $D=0.33 \mathrm{~m}$ and the thickness is $0.01 \mathrm{~m}$. The rotor has been specially designed for the ventilation and cooling applications of a generator, such as the ones used on vertical axis wind turbines (VAWT). 


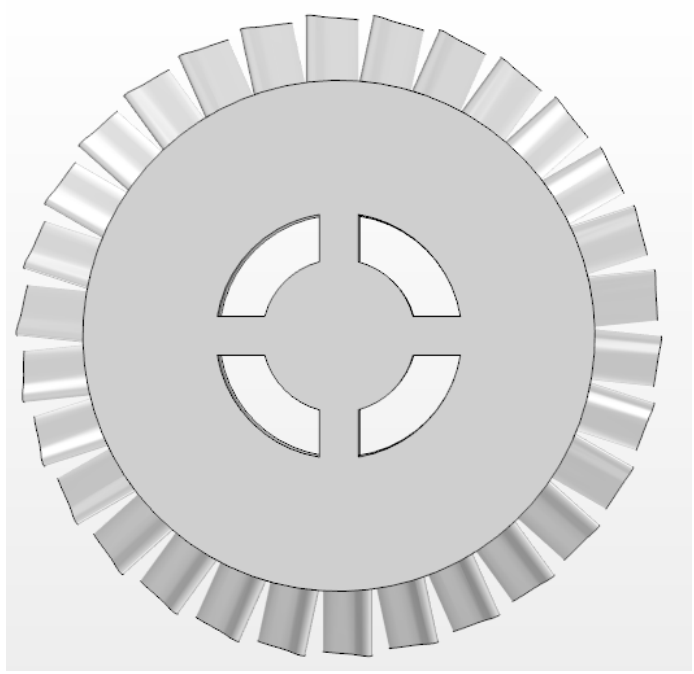

(a) Front view

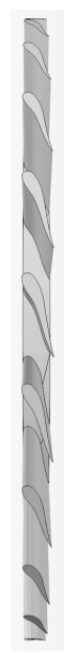

(b) Side view

Figure 2. Axial flow fan rotor geometry.

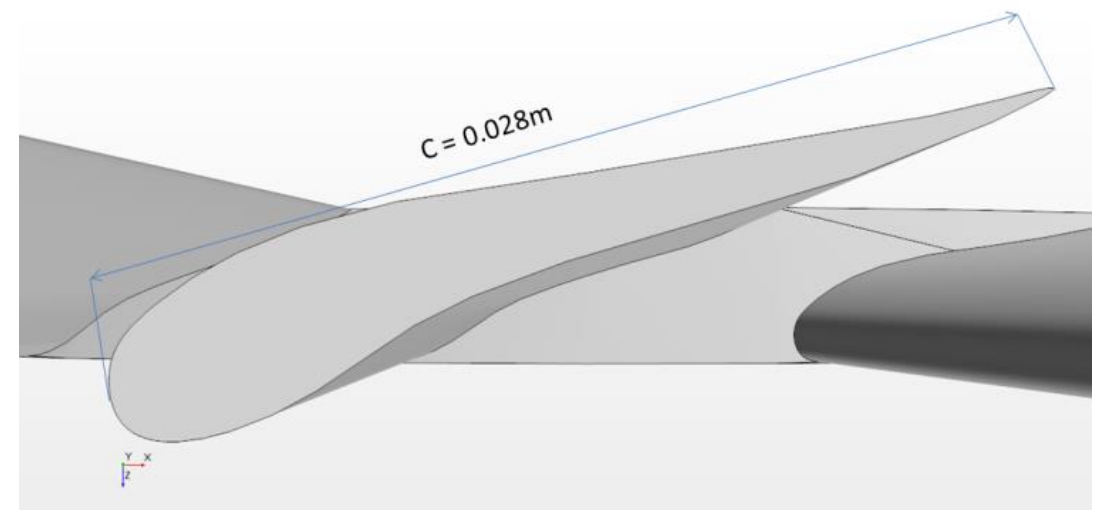

Figure 3. NACA airfoil based blade.

The problem involves an axial fan rotor whose axis and 30 equally spaced blades are rotating at 200 $\mathrm{rpm}$ while the external body remains stationary. Air enters through the inlet at $5 \mathrm{~m} / \mathrm{s}$ and exits through a pressure boundary in the outlet. Fig. 4 represents the boundary conditions and the dimensions of the computational domain used in this study. The axial fan rotor has been placed in a circular cylinder with length three times the rotor diameter.

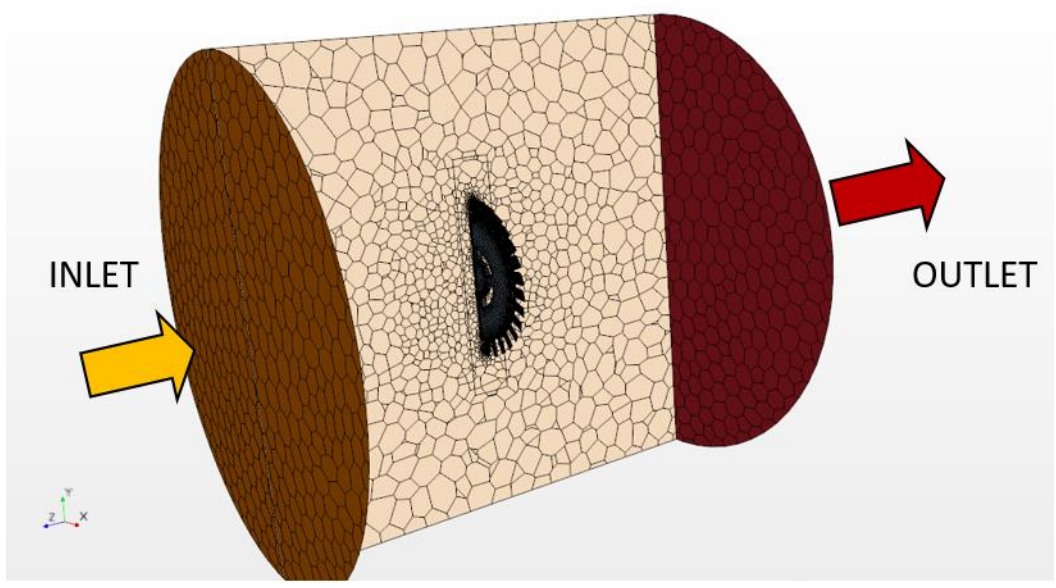

Figure 4. Computational domain 


\subsection{Mesh Distribution and Physics Models}

The grid of the current model consists of two regions: Fluid and Rotation. However, only one continuum is needed for the simulation since two regions contain the same fluid. The physical models for this continuum are specified in Table 1.

Table 1. Physical characteristics

\begin{tabular}{ll}
\hline Properties & Models \\
\hline Time & Unsteady \\
Fluid & Air Standard Conditions \\
Flow & Incompressible flow \\
Viscose regime & Turbulent \\
Reynolds Averaged Turbulence model & K-Epsilon \\
\hline
\end{tabular}

In this work, two interfaces between the Fluid and Rotating regions have been created. This process involves a creation so-called "in-place" interfaces between the default wall boundaries enclosing each region (Fig. 5). The transient Rigid Body Motion model to solve the problem has been used in these simulations.

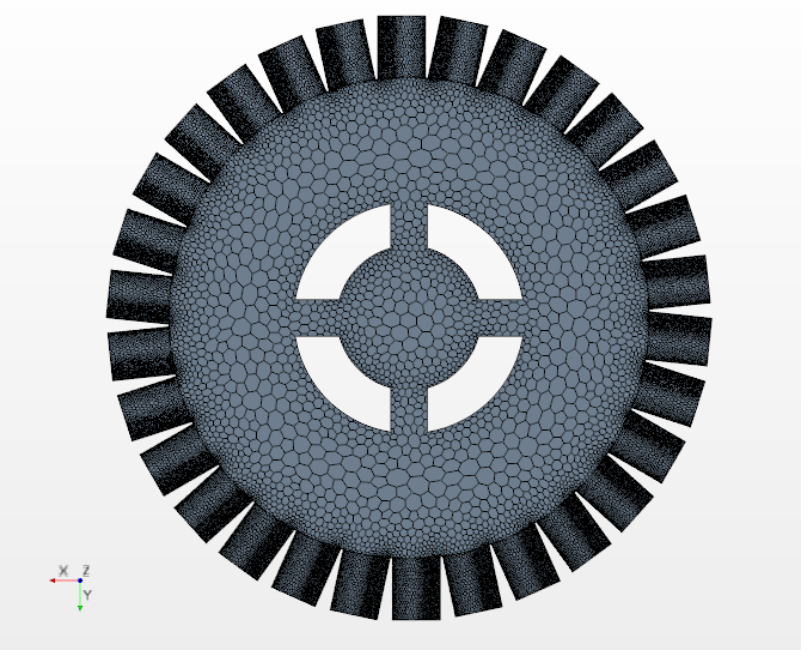

(a) Front view

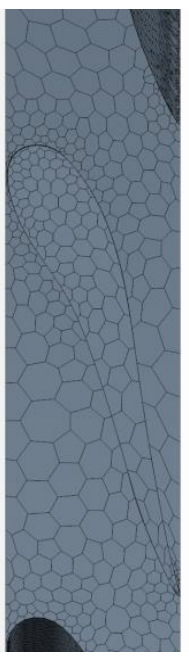

(b) Side detailed view

Figure 5. Meshed rotor views.

\section{RESULTS and DISCUSSION}

Pressure and velocity field around the rotor have been studied and computed during the period of 20 seconds, which corresponds to 66.67 revolutions of the rotor. Fig. 6(a) illustrates the absolute pressure distribution around the airfoils of the rotor. This figure shows the difference in pressure between the upper and the lower part of the blade. Fig. 6(b) represents the axial velocity distribution according to the reference frame axis coordinate used for the rotor rotation. The flow is completely attached in the upper part of the blade. However, some flow separation has been detected in the lower part of the airfoil. Some negative values of the axial velocity have been found in the lower surface of the airfoils, which means that there exists a reverse flow in that region. The separation of the flow could be the result of the adverse pressure gradient formed in the convex part of the lower surface of the airfoil, close to the region with the maximum camber. For Fig. 6(a, b), it should be noted that the air flow has been considered to come with a certain angle to the airfoil, that indeed differs from the real experimental conditions, which uses a direct horizontal flow. If inlet and outlet are taken under air conditions and rotating body are defined fan for $300 \mathrm{rpm}$ angular velocity, velocity and pressure fields distribution around the rotor are shown as in Fig.7(a, b). Velocity magnitude increases when it comes closer to the rotor unit. The maximum pressure has been obtained on blade points, which are the first meeting locations of the blade. 


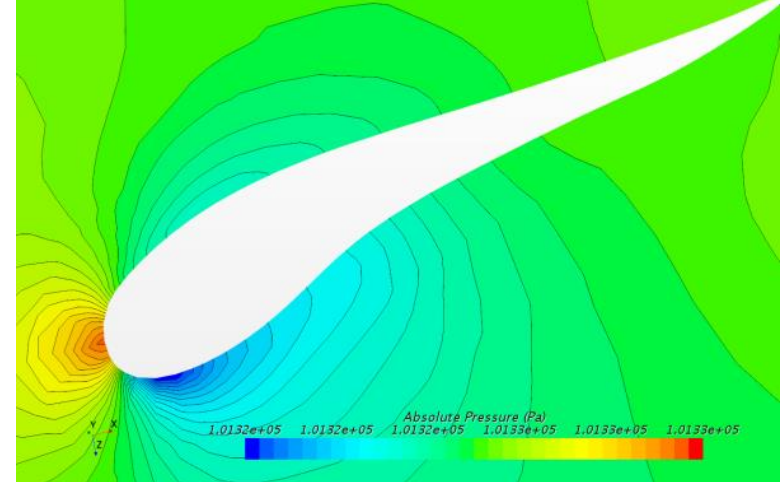

(a) Absolute pressure field

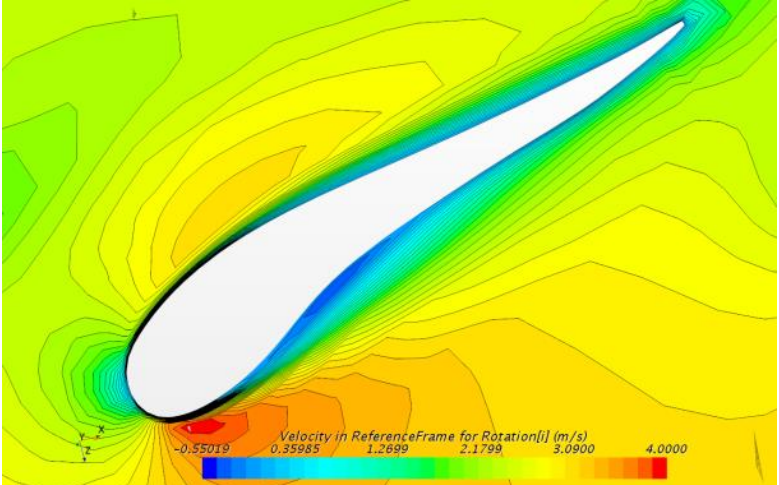

(b) Axial velocity field

Figure 6. Pressure and velocity fields distribution around a rotor blade.

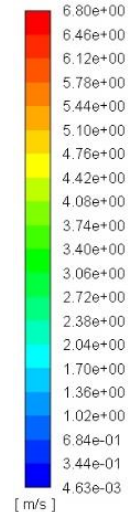

(a) Velocity field

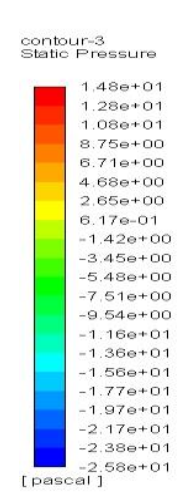

(b) Static Pressure field

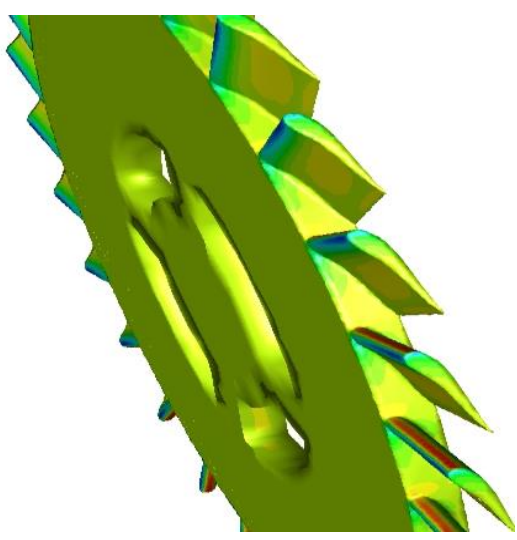

Figure 7. (a) Velocity and (b) pressure field distribution around rotor and its blades.

Note that the pressure values are positive on the upper part of the section, whereas it turns to slightly negative for lower part. The velocity values change between $1.02 \mathrm{~m} / \mathrm{s}$ and $4.76 \mathrm{~m} / \mathrm{s}$ as shown in Fig. 7(a). In addition, a pressure value of 2.65 Pascal comes to the front of the fan. Mainly a deviation between 14 Pascal and -2.6 Pascal occurs for the overall fan (Fig. 7(b)). Fig. 8(a-f) shows the representative findings for inlet/outlet values of static and total pressures and velocity distributions.

According to Fig. 8(a, b), the inlet can have static pressures up to 4.7 Pascal, the outlet can have the pressure values $P=2.65$ Pascal, which is slightly below of the inlet. Note also that the general pressure is around 0.6 Pascal at the overall surface of rotor and it is slightly higher at the outlet. In terms of total pressure, one reads as slightly lower values than the static pressures as seen in Figs. 8(c, d). Pressures of outlet are more regular than inlet. In the case of velocity distribution, the maximal inlet value is located around the front of blades as $1.7 \mathrm{~m} / \mathrm{s}$, whereas the outlet distribution has the value of $2.04 \mathrm{~m} / \mathrm{s}$, which is a good value for the cooling aim. Note that the location of rotor in generator is at first stage and next to $1,5 \mathrm{~mm}$ from stator body. Reference points were selected both side next to $1,5 \mathrm{~mm}$ of the first rotor.

In Fig. 9(a, b), the findings for velocity and power are plotted. The velocity amount increases linearly for the inlet and outlet regions of the fan. It proves that the fan operates properly since the velocity of outlet becomes higher than the inlet velocity. Strictly speaking, the velocities get value from $0.79 \mathrm{~m} / \mathrm{s}$ to $3.24 \mathrm{~m} / \mathrm{s}$. In addition, the difference between two plots increases by the angular velocity. It means 
that higher angular velocities beyond $1000 \mathrm{rpm}$ yields to better velocity increase at the outlet with a contribution of $0.5 \mathrm{~m} / \mathrm{s}$.

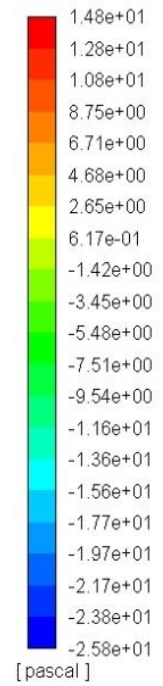

(a) Static pressure on reference point for Inlet

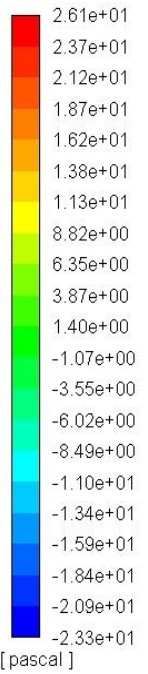

(c) Total pressure on reference point for inlet

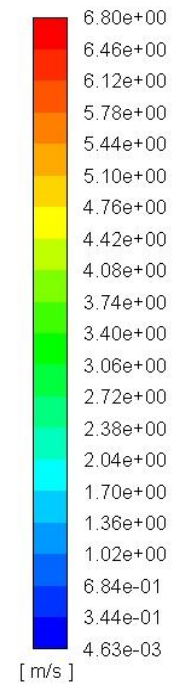

(e) Velocity Distrubutionon Reference point for inlet

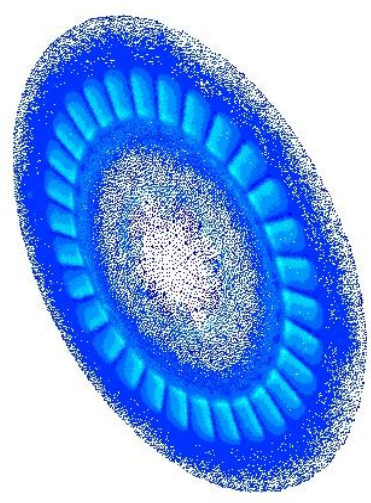

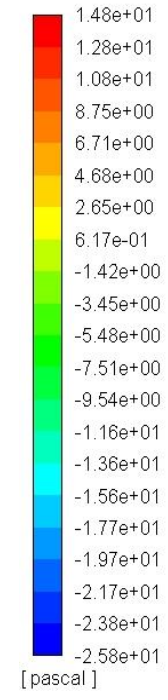

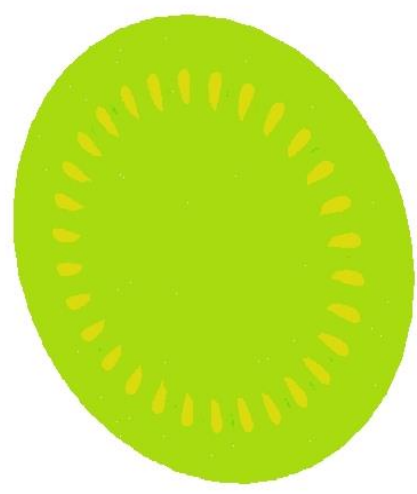

(b) Static pressure on reference point for Outlet
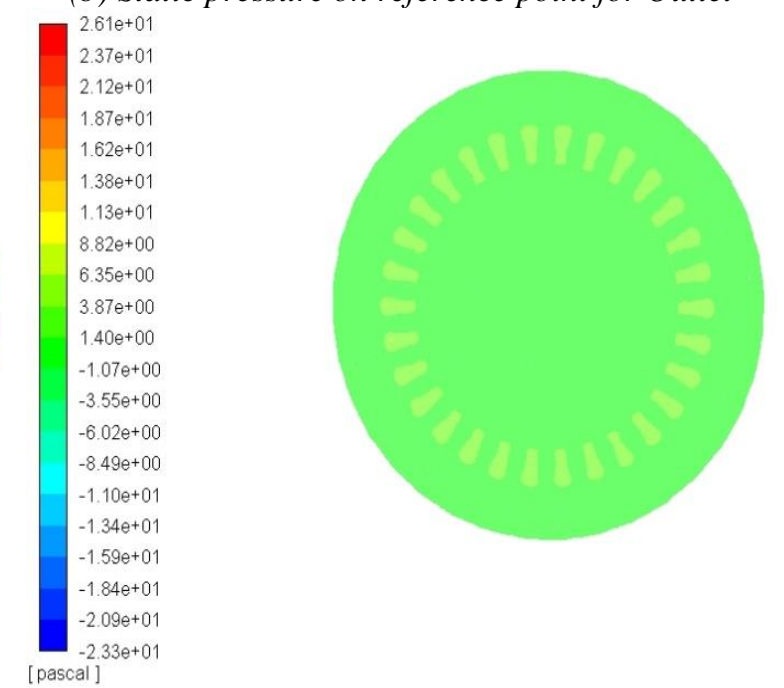

(d) Total pressure on reference point for outlet

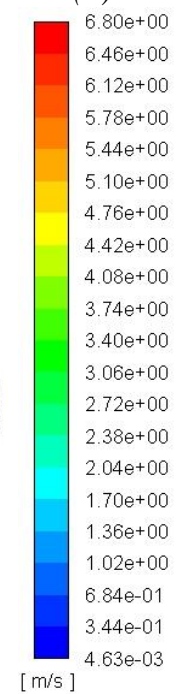

(f) Velocity Distrubutionon Reference point for outlet

Figure 8. Pressure and velocity fields distribution around reference plane 


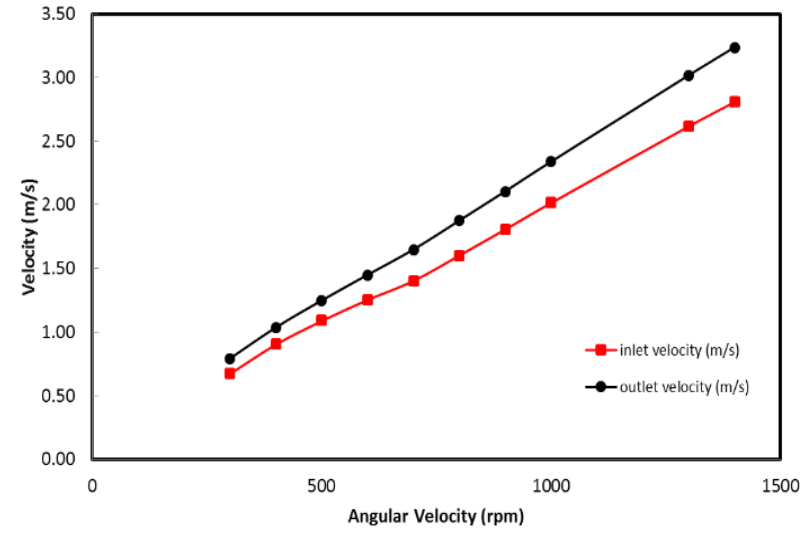

(a) Air Velocity Graphics

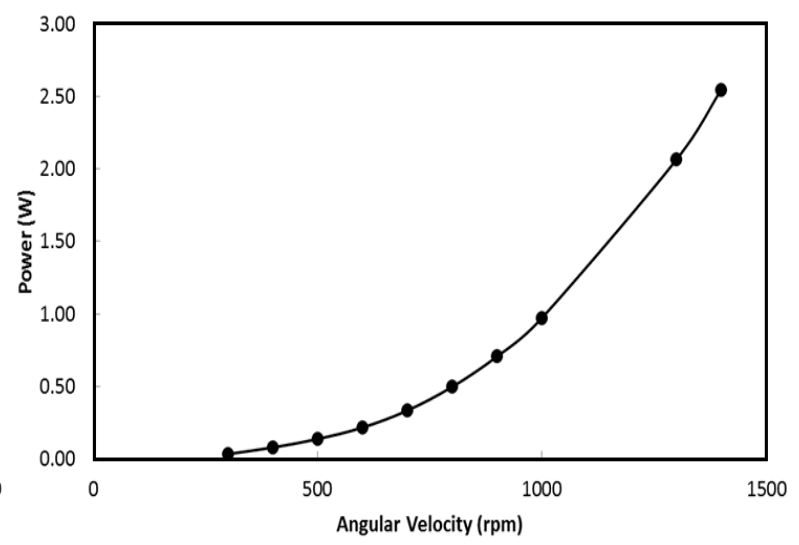

(b) Fan Power Graphics

Figure 9. Plots of (a)air velocity and (b)power with respect to the angular velocity

For all those ANSYS Fluent simulations, the boundary conditions for the inlet and outlet are taken as stagnant, zero velocity and pressure. According to Fig. 9(b), the outlet power for different angular velocities starts from $0.035 \mathrm{~W}$ and increases up to $2.549 \mathrm{~W}$ for $1400 \mathrm{rpm}$ of the axial fan.

\section{CONCLUSIONS}

Numerical simulations of a single stage axial fan rotor at Reynolds number of $\operatorname{Re}=1.110^{5}$ based on the rotor diameter have been performed to obtain the detailed flow field of the centrifugal fan rotor. The work has been mainly focused on the rotor configuration and the modeling of aerodynamic behavior of the blades. In addition, the rotor of the axial fan has been designed according to the requirements of the application to the cooling system of a vertical axis wind turbine. Preliminary results show a good aerodynamic behavior of the rotor at the studied conditions of velocity in the inlet of $5 \mathrm{~m} / \mathrm{s}$ and rotor rotational speed of $200 \mathrm{rpm}$. However, some improvements in the airfoil design of the rotor must be done to avoid separation of the flow in the lower surface of the airfoil of the blade, when the attach angle changes from horizontal direction. At the second case, axial fan was investigated in the stagnant air condition for various angular velocities in a rotating regime. Velocity and power concepts have been studied and they are found as $0.79 \mathrm{~m} / \mathrm{s}-3.24 \mathrm{~m} / \mathrm{s}$, and $0.035 \mathrm{~W}$ to $2.549 \mathrm{~W}$ for 300 to $1400 \mathrm{rpm}$, respectively. As a future work, more computations should be carry out at different Reynolds numbers to check the aerodynamics performance of the rotor. Also, convenient calculations should be done for the design and validations of the axial fan shroud.

\section{Acknowledgment}

The authors are grateful to European Union Ministry of Turkey, National Agency of Turkey for the support of this project under the Project Code: 2015-1-TR01-KA203-021342 entitled Innovative European Studies on Renewable Energy Systems. The funding from the Government of the Basque Country and the University of the Basque Country UPV/EHU through the SAIOTEK (S-PE11UN112) and EHU12/26 research programs are also acknowledged.

\section{REFERENCES}

[1] Prachar, A. Comparison of axial fan rotor experimental data with CFD simulation. Acta Polytech 2016; 56(1): 62-66; DOI: 10.14311/AP.2016.56.0062.

[2] Matai, R, Yavuzkurt, S. Evaluation of Effects of Different Design Parameters on Axial Fan Performance using Cfd. In: ASME Turbo Expo 2015: Turbine Technical Conference and Exposition: 15-17 June 2015, ASME, Montreal, Qebec, Canada.

[3] Masi, M , Castegnaro, S , Lazzaretto, A. A Criterion for the Preliminary Design of High-Efficiency TubeAxial Fans. In: ASME Turbo Expo 2016: Turbomachinery Technical Conference and Exposition: 13-17 June 2016, ASME, Seul, South Korea. 
[4] Gör, H, Kurt, E. Preliminary studies of a new permanent magnet generator (PMG) with the axial and radial flux morphology. International Journal of Hydrogen Energy 2016; 41(17): 7005-7018.

[5] Çelik, E, Gör, H, Öztürk, N , Kurt, E. Application of artificial neural network to estimate power generation and efficiency of a new axial flux permanent magnet synchronous generator. International Journal of Hydrogen Energy 2017; 42(28); 17692-17699.

[6] Pelletier, D, Schetz, J. Finite-Element Navier-Stokes Calculation of 3-Dimensional Turbulent-Flow Near a Propeller. Aiaa J. 1986; 24(9); 1409-1416.

[7] Yoon, S, Schetz, J. Numerical Navier-Stokes Solutions of High-Speed Propeller Flows. J. Propul. Power 1988; 4(4); 291-292.

[8] Twizell, E, Bright, N. Numerical Modeling of Fan Performance. Appl. Math. Model. 1981; 5(4); $246-250$.

[9] Wright, T, Gerhart, PM. Fluid Machinery. Application, Selection, and Design. CRC Press (second edition ed.), 2009.

[10] Lazari, A, Cattanei, A. Design of Off-statistics Axial-flow Fans by Means of Vortex Law Optimization. Journal of Thermal Science 2014; 23(6); 505-515.

[11] Heo, S, et al. Development of high-performance and low-noise axial-flow fan units in their local operating region. Journal of Mechanical Science and Technology 2015; 29(9); 3653-3662.

[12] Valbracht, RA. Laboratory methods of testing induced flow fans for rating, the development of amca standard 260-07. In: AMCA International Engineering Conference, 2008, Las Vegas, NV, USA.

[13] ASME. ASME-PTC 11fans. 2008.

[14] Castegnaro, S. Fan Blade Design Methods: Cascade Versus Isolated Airfoil Approach - Experimental and Numerical Comparison. In: ASME Turbo Expo 2016: Turbomachinery Technical Conference and Exposition: 13-17 June 2016, ASME, Seul, South Korea.

[15] Carolus, T H, Starzmann, R. An Aerodynamic Design Methodology for Low Pressure Axial Fans with Integrated Airfoil Polar Prediction. In: ASME 2011 Turbo Expo: Turbine Technical Conference and Exposition: 06-10 June 2011, ASME, Vancouver, British Columbia, Canada.

[16] Diangui, H. 3D optimum design of reversible axial flow fan with S-type blade. Chin. J. Mech. Eng. 2005; 41(12); 182-185. 